\title{
Tropospheric Delay Estimation for Pseudolite Positioning
}

\author{
Jianguo Jack Wang, Jinling Wang \\ School of Surveying and Spatial Information Systems, The University of New South Wales, Sydney, NSW 2052, Australia \\ e-mail: jianguo.wang@student.unsw.edu.au) _ Phone: +61-2-9385 4185 Fax: +61-2-9313 7493
}

\section{David Sinclair, Leo Watts}

QASCO Surveys Pty. Limited, 41 Boundary St. South Brisbane, Australia

\section{Hung Kyu Lee}

Electronics and Telecommunications Research Institute (ETRI), Rep. of Korea

Received: 15 Nov 2004 / Accepted: 12 July 2005

\begin{abstract}
Pseudolites, ground-based GPS signal transmitters, can significantly enhance the GPS satellite geometry or can even be an independent positioning system. However, as pseudolites are very close to the receivers, error effects are different from the traditional GPS and should be considered and modeled in a different way. Tropospheric delay is one of the largest error sources in pseudolite positioning, as pseudolite signal propagates through the lower troposphere which is very difficult to be modeled due to spatial variations in atmosphere. The objective of this research is to analyse pseudolite tropospheric delay modelling methods and to select the optimal tropospheric delay models for different applications.
\end{abstract}

Several methods to estimate the tropospheric delay for pseudolite positioning are introduced and compared. One approach is to utilize single-differenced GPS tropospheric models. Another one is to compute the tropospheric delay as a function of the local refractivity along the pseudolite signal path. The ratio method used for Electronic Distance Measurement (EDM) can also be applied to estimate tropospheric delay.

Experiments with simulation and real flight test data are conducted in this study to investigate the proposed methods. The advantages and limitations of each method are analysed. The mode defined by RTCA and its modification are suitable for a low elevation and short range application, such as LAAS and local ground based applications. Models derived from single-differenced NMF and Saastamoinen models perform well in long range and high elevation but have a big bias in low elevation. And the model derived from the Hopfield model performs relatively well in all the range and elevation.
Key words: Pseudolite, tropospheric delay, modelling

\section{Introduction}

GPS has been widely used for precise positioning and navigation applications. However, the accuracy, availability, reliability and integrity of GPS positioning solutions heavily depend on the number and geometric distribution of GPS satellites being tracked. Furthermore, GPS cannot be used without line-of-sight signals between the GPS satellites and a receiver. Pseudolites, which are ground-based GPS-like transmitters, can significantly enhance the satellite geometry or can even construct an independent positioning system.

Compared with GPS satellites, pseudolites are very close to the receivers. Therefore there are many effects that have to be considered and modeled in a different way. Whereas for GPS satellite signals most of the error sources can be eliminated by difference techniques, few of the error sources of the pseudolite signals can be eliminated or mitigated from the analogous approach. The practical solution for pseudolites is to estimate them accurately.

Tropospheric delay can be the largest error source of pseudolite signal as it propagates through the lower troposphere which is very difficult to model due to spatial variations in atmospheric pressure, temperature and humidity. In general, the truth model of tropospheric delay is a function of temperature, atmospheric pressure, relative humidity, elevation angle and range. As 
atmospheric parameters are normally taken at the reference station, there is always bias when estimating the parameters along the path of signal propagation. This research is to model the tropospheric delay for pseudolite positioning, and to analyze the advantages and limitations of each method by conducting experiments of simulation and real flight data processing.

There are a few tropospheric delay models introduced for pseudolite positioning, such as RTCA model (RTCA, 2000) and Biberger model (Biberger et al., 2003) regarding LAAS (Local Area Augmentation System) for precise aircraft approaching and landing. These two models are suitable for a low altitude measurement, but there are big biases when the altitude is comparable with the scaled heights for the models. The Bouska and Raquet model (2003) and another model derived from the Hopfield model are also investigated in this paper. The above four models are based on a method that computes the tropospheric delay as a function of the local refractivity along the pseudolite signal path.

Another method introduced in this paper is to estimate pseudolite tropospheric delay using single-differenced GPS tropospheric models. As the performance of tropospheric propagation delay prediction models used for GPS typically degrades at very low elevation angles, the models were designed to be used above a limiting elevation angle. Pseudolite tropospheric delay equations derived from single-differenced NMF (Niell Mapping Function) and Saastamoinen models can be applied when the elevation angle is higher than 4 and 10 degrees respectively.

This research reveals the importance of the tropospheric delay modelling for pseudolite positioning, and analyses the performance of the six models proposed for pseudolite tropospheric delay estimation. The advantages and limitations of each model are investigated by analysing simulation results from different aspects. Flight test data were processed to verify the results.

\section{Tropospheric Delay Estimation Methods}

Common GPS tropospheric delay models are not sufficient for pseudolite positioning. In contrast to the DGPS principle, the signal path from the pseudolite transmitting antenna to the reference station and the signal path from the pseudolite to the rover receiver pass through very different parts of the troposphere. Whereas the distance between pseudolite and reference station is constant, short and the signal runs close to the surface, the distance between pseudolite and rover receiver varies rapidly with time. Even more momentousy, the quantity of tropospheric delay errors is strongly dependent on vertical differences. Therefore, a powerful modeling of the tropospheric effects has to be accurately considered in the pseudolite error model.

Three methods are suggested here to estimate tropospheric delay for pseudolite ranging. One is to compute the tropospheric delay as a function of the local refractivity by integrating local refractivity along the pseudolite signal path. For simplicity, it is called the integration method. Normally, empirical models are employed to represent local refractivity.

Another method utilizes single-differenced GPS tropospheric delay models. The models of GPS tropospheric delay are relatively better developed and can reach very high accuracy. It is reasonable to derive models for pseudolite from them. However, as the performance of GPS tropospheric delay models degrades at very low elevation angles, models derived from these models could have a big bias in a low elevation angle, though they perform well in a long range and high elevation angle. Thus, there is a limited elevation angle of signal slant path for the models derived with singledifferenced method.

For short distance measurement at similar altitude, the length ratio method used for Electronic Distance Measurement (EDM) (Rueger, 1996) can be applied to correct pseudolite tropospheric delay. It is assumed that all distance measurements have proportional atmospheric (refractive index) effects. A common scale parameter is assigned to each measurement. No measurements of atmospheric parameters are required. Scales should be adjusted when the receivers are not at the same height.

In this paper the models using the first two methods, integration and single-difference method, are analysed with simulation and flight test data. Four models are applied with the integration method and two models with the single-difference method.

\subsection{Models of Integration Method}

RTCA has defined the tropospheric delay model for LAAS (RTCA, 2000). The tropospheric correction consists of a dry (hydrostatic) and a wet component.

$$
\Delta_{\text {trop }}=\Delta_{d r y}+\Delta_{w e t}
$$

The dry and wet components are to be determined separately by Equation (2). The “*” in Equation (2) is to be read as the parameter for dry and wet respectively.

$$
\Delta_{*}=10^{-6} \cdot N_{*, 0} \cdot R_{\text {rov }} \cdot\left(1-\frac{h_{\text {rov }}-h_{P L}}{h_{*, 0}}\right)
$$


The rover receiver height $h_{\text {rov }}$ and the pseudolite height $\mathrm{h}_{\mathrm{PL}}$ in Equation (2) declare the importance of the vertical distance for tropospheric modeling while $\mathrm{R}_{\text {rov }}$ is the slope distance between rover receiver and pseudolite. Meteorological data is used to determine the refraction index $\mathrm{N}_{*}$, which is defined by Equation (3). $h_{*, 0}$ is a fixed scaled height for the model which is $42,700 \mathrm{~m}$ for the hydrostatic component, and $12,000 \mathrm{~m}$ for the wet component. These heights are arbitrarily defined as the upper boundaries for tropospheric refraction.

$$
\begin{aligned}
& N_{d r y}=77.6 \cdot \frac{P}{T} \text { and } \\
& N_{\text {wet }}=22770 \cdot \frac{f}{T^{2}} \cdot 10 \frac{7.4475 \cdot(T-273)}{T-38.3}
\end{aligned}
$$

In Equation (3) $T$ is the temperature, $f$ is the relative humidity and $P$ is the atmospheric pressure sampled on the spot. These parameters then have to be reduced at sea level before they can be used in Equation (2).

Werner (Biberger et al., 2003) proposed some modifications to the RTCA model, which leads Equation (2) to be substituted by Equation (4):

$$
\begin{aligned}
\Delta_{*} & =10^{-6} \cdot N_{*, 0} \cdot R_{\text {rov }} \cdot \\
& \left(1-2 \cdot \frac{h_{r o v}-h_{P L}}{h_{*, 0}}+2 \cdot \frac{h_{r o v}^{2}+h_{r o v} \cdot h_{P L}+h_{P L}^{2}}{h_{*, 0}}\right)
\end{aligned}
$$

The tropospheric delay model proposed by Bouska and Raquet (2003) is evidently derived from the Hopfield model, with a modified surface height. The $\mathrm{N}_{*}$ in Equation (5) is the refraction index at the height of pseudolite while $\mathrm{N}_{*, 0}$ in the Equations (2), (4) and (6) is refraction index at sea level.

$$
\begin{aligned}
\Delta_{*} & =2 \cdot 10^{-5} \cdot N_{*} \cdot R_{\text {rov }} \cdot \\
& \left(1-\left(1-\frac{h_{\text {rov }}-h_{P L}}{h_{*}}\right)^{5}\right) \cdot \frac{h_{*}}{h_{\text {rov }}-h_{P L}}
\end{aligned}
$$

where $h_{*}=h_{*, 0}-h_{P L}$ and $N_{*}(h=0)=N_{*, 0}$.

Another model directly derived from the Hopfield model is Equation (6), which is an integral from the surface of the sea level (Hofmann-Wellenhof, 2000).

$$
\begin{aligned}
\Delta_{*} & =2 \cdot 10^{-5} \cdot N_{*, 0} \cdot R_{\text {rov }} \\
& \left(\left(1-\frac{h_{r o v}}{h_{*, 0}}\right)^{5}-\left(1-\frac{h_{P L}}{h_{*, 0}}\right)^{5}\right) \cdot \frac{h_{*, 0}}{h_{\text {rov }}-h_{P L}}
\end{aligned}
$$

It is noticed that Equations (5) and (6) are identical if the Hopfield model is applied for calculating $N_{*, 0}$, and different if height-dependent values of pressure, temperature and humidity are used. These four models listed above are based on the integration method that computes the tropospheric delay as a function of the local refractivity along the pseudolite signal path.

\subsection{Models of Single-differenced Method}

Models derived from the single-differenced GPS tropospheric delay are based on the concept described in Figure 1. The GPS tropospheric delays are calculated from the rover receiver and pseudolite to a GPS satellite in the same line with them using the well-known NMF and Saastamoinen models. The tropospheric delay from the pseudolite to the rover receiver is the difference of the two values.

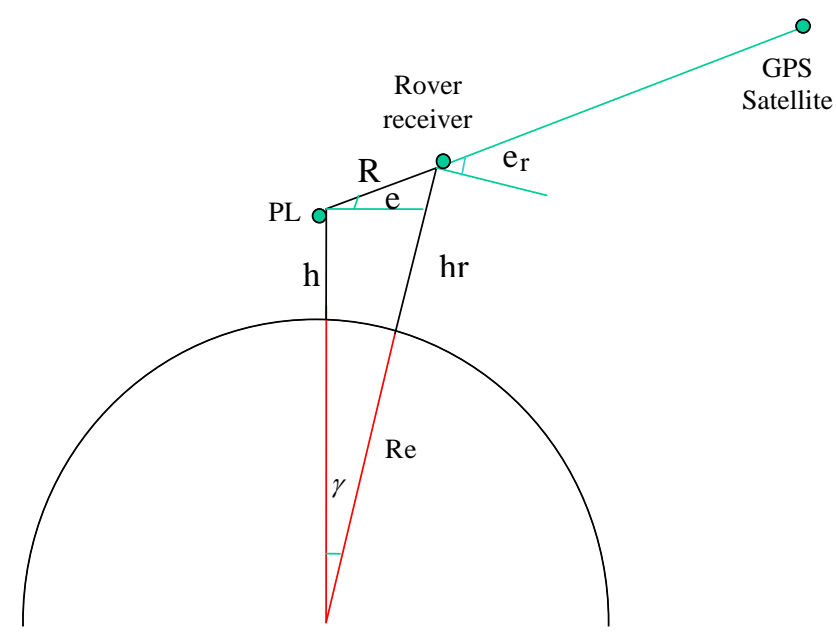

The Earth centre

Fig. 1 Concept of single-differenced method

Figure 1 depicts the relation of the positions of pseudolite, the satellite and rover receiver. Equations (7) and (8) are the formulas to calculate the height of the rover receiver and elevation angle from it to the supposed GPS satellite used for NMF or Saastamoinen tropospheric delays models.

$$
\begin{aligned}
h_{r} & =\sqrt{\left(R_{e}+h\right)^{2}+R^{2}-2 \cdot\left(R_{e}+h\right) \cdot R \cdot \cos (e+\pi / 2)} \\
& =\sqrt{\left(R_{e}+h\right)^{2}+R^{2}+2 \cdot\left(R_{e}+h\right) \cdot R \cdot \sin e}
\end{aligned}
$$

and

$$
\begin{aligned}
e_{r} & =e+\gamma \\
& =e+\arcsin \left(R \cdot \sin (e+\pi / 2) /\left(R_{e}+h_{r}\right)\right) \\
& =e+\arcsin \left(R \cdot \cos e /\left(R_{e}+h_{r}\right)\right)
\end{aligned}
$$


The Saastamoinen model (Remomd, 2000) can precisely estimate GPS tropospheric delay for elevation angle larger than 10 degrees. This model is expressed as:

$$
\begin{aligned}
\Delta^{\text {trop }} & =\frac{0.002277}{\cos Z} . \\
& {\left[p+\left(\frac{1255}{T}+0.05\right) \cdot e-B \cdot \tan ^{2} Z\right]+\delta R }
\end{aligned}
$$

where $\boldsymbol{Z}$ denotes the zenith angle and $\boldsymbol{p}$ is the atmospheric pressure and $\boldsymbol{e}$ is water vapour pressure in millibar; $\boldsymbol{T}$ is the temperature in Kelvin. $\boldsymbol{B}$ and $\boldsymbol{\delta} \boldsymbol{R}$ are two correction terms, one being dependent on height of the observing site and the latter on the height and zenith angle. They can be interpolated from the tables provided by a refined model.

The NMF (A.E.Niell, 1996) claims high accuracy estimation for elevation angles larger than 4 degrees. The coefficients in the model depend on the latitude and the height at the observing site and on the day of the year. These two models are applied with the singledifferencing method to calculate the tropospheric delay for pseudolites.

\section{Simulation Results Analysis}

The six models introduced above are programmed under standard atmosphere model $\left(\mathrm{P}_{0}=1013.25 \mathrm{mb}, \mathrm{T}_{0}=18^{\circ} \mathrm{C}\right.$, $\mathrm{H}_{0}=50 \%$ ) at sea level. An analysis is conducted by comparing them from different aspects.

\subsection{Comparing the Models with Different Elevation Angle}

Figures 2 and 3 show the tropospheric delays calculated from the six models in a range of $5 \mathrm{~km}$ with 0 and $1 \mathrm{~km}$ reference heights respectively. The elevation angle changes from 0 to 90 degrees. As mentioned above, the values given by the Niell model with elevation angles less than 4 degrees and by Saastamoinen model with elevation angle less than 10 degrees should be ignored. However the figures show that the values given by the Niell model with elevation angle less than 4 degrees do not drift much.

It can be seen from Figures 2 and 3 that the difference of tropospheric delays estimated with different models can reach more than $30 \mathrm{~cm}$ in a range of $5 \mathrm{~km}$. Delay from Hopfield model is larger than that from Bouska model when reference height is higher than zero, and they are identical when the reference height is zero. The delays of Niell, Saastamoinen and Bouska models (and Hopfield model when reference height is zero) are similar when the elevation angle is big, but the estimation of TRCA and modified TRCA models deviate from them at large elevation angles.

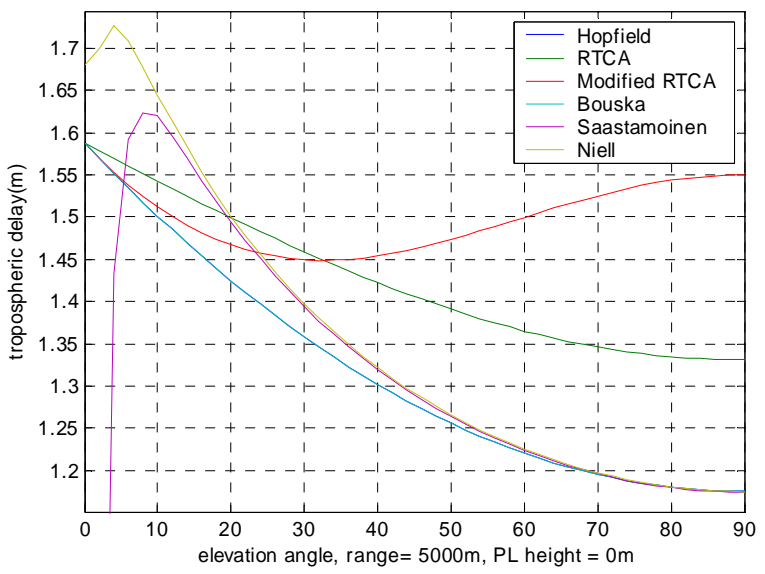

Fig. 2 Changes with elevation angles $(\mathrm{h}=0 \mathrm{~m})$

The estimations of the Bouska, TRCA and modified TRCA models are the same when the elevation angle is zero. Provided that refractivity at the same height changes little, it is reasonable to believe that the tropospheric delays estimated by models of integration method are accurate at low elevation angle.

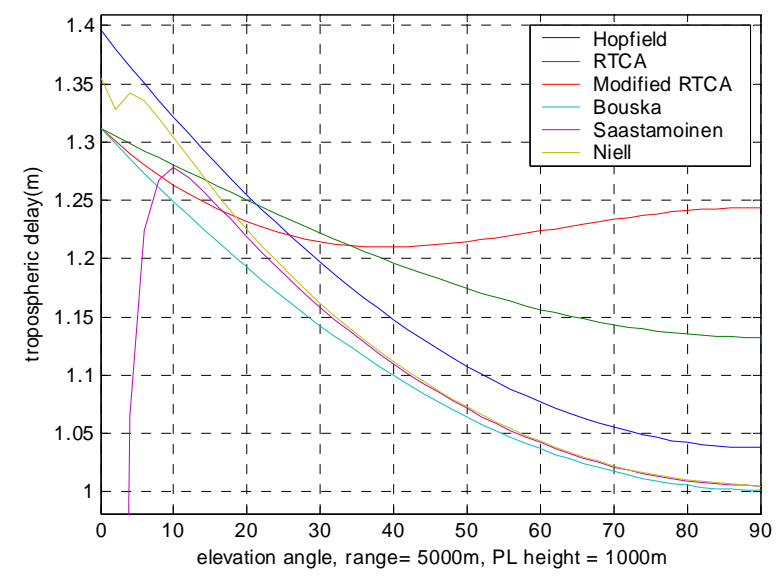

Fig. 3 Changes with elevation angles $(\mathrm{h}=1000 \mathrm{~m})$

\subsection{Comparing the Models with Different Reference Height}

Figures 4 and 5 show the tropospheric delays calculated from the six models in a range of $3 \mathrm{~km}$ with a 10 and 90 degrees elevation angle, respectively. The reference height changes from 0 to 2000 meters.

These figures show that the differences of the estimations between the models become smaller as the reference height increases, especially for a low elevation angle. The estimation of TRCA and modified TRCA model are good 
with a small elevation angle (Figure 4) but not accurate with a large elevation angle (Figure 5). The estimation of Niell and Saastamoinen models are very similar at different heights and identical at high elevation angles. The line generated by the Hopfield model is less curvy than the others.

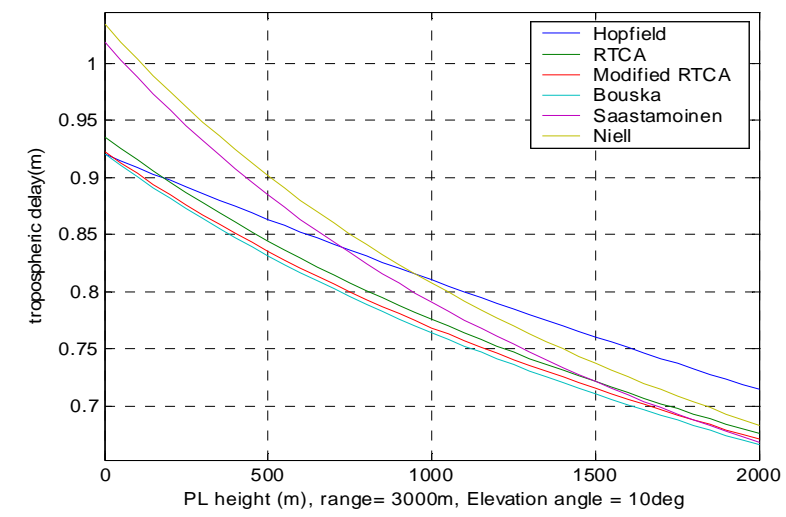

Fig. 4 Changes with reference height (elevation angle is 10 degrees)

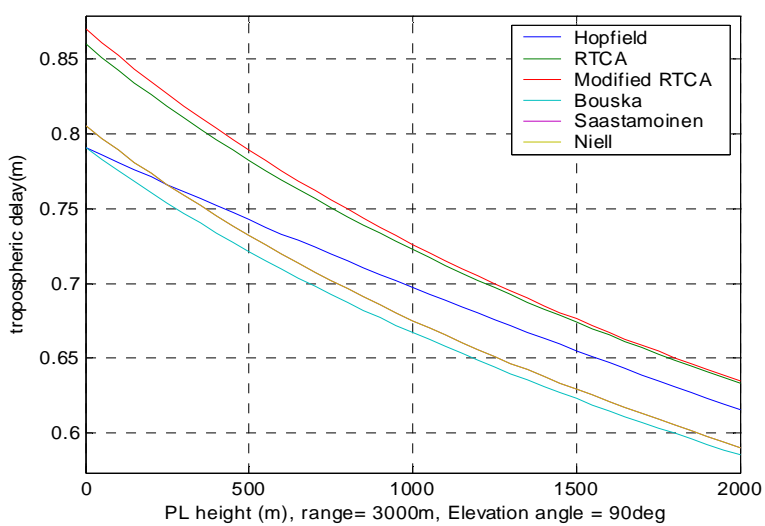

Fig. 5 Changes with reference height (elevation angle is 90 degrees)

In general, it can be concluded from the simulation results that there are observable differences of tropospheric delays estimated with different models. Each model has its strong and weak points. The TRCA and modified TRCA models can be used in the applications with small height difference, such as aircraft landing and land-based applications. However, they are not suitable for large height difference, such as precise airborne georeferencing. Niell and Saastamoinen models are reliable at high elevation angle but unreliable at very low elevation angle, as shown in Figures 2 and 3 . The Hopfield and Bouska models perform relatively stable over the whole range of elevation angle although there is a reference height dependent bias between them. It can be seen from Figures 2 and 3 that the estimation of Bouska model is approaching to that of the RTCA at zero degree and to that of the Niell and Saastamoinen models at 90 degrees. It indicates that this model should have the smallest bias among all the models in the whole range of elevation angle.

\section{Flight Test Data Analysis}

Flight tests were conducted in April 2003 at the Wedderburn Airfield, Sydney, Australia. Figure 6 shows the sky plot of the GPS satellites and Figure 7 shows the relative height, distance and horizontal trajectory of the aircraft during the period used for data processing.

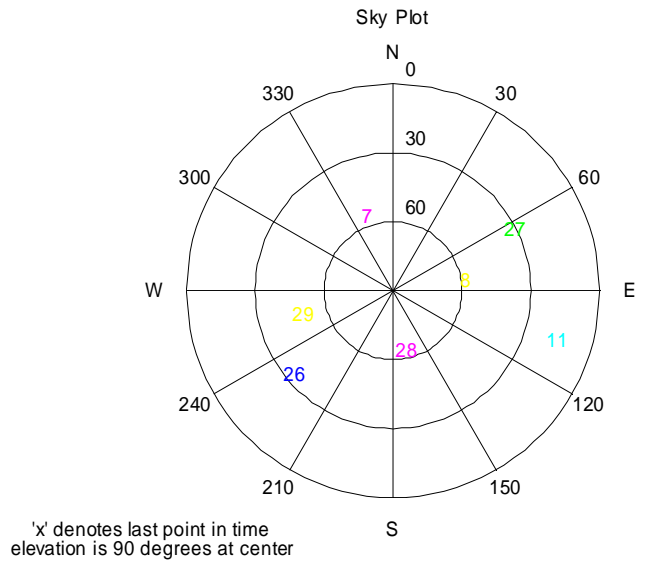

Fig6 Sky plot of GPS satellites
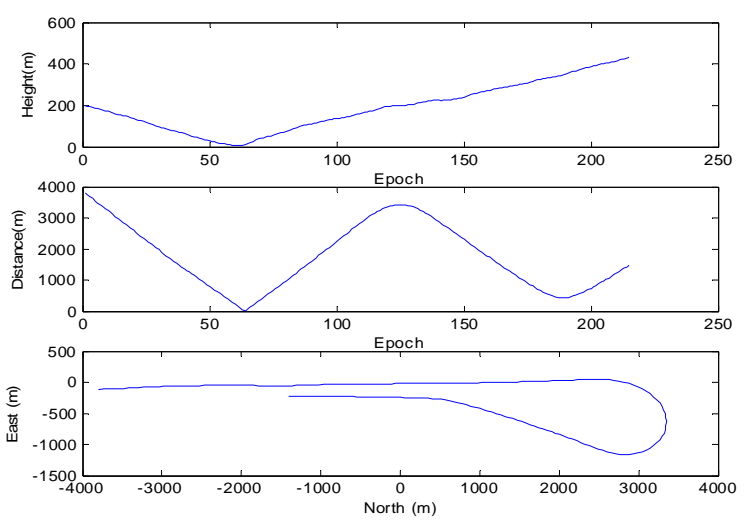

Fig. 7 Flight trajectory used for processing

The pseudolite signal was generated by a Spirent Communications GSS4100P single-channel signal generator pulsing at a $1 / 11$ duty cycle, with a $10 \mathrm{MHz}$ oven-controlled crystal oscillator frequency reference. The reference station consisted of a NovAtel Millennium receiver with Leica AT504 choke-ring antenna. Note that use of the choke-ring was for the mitigation of GPS/pseudolite multipath. The airborne system comprised two GPS/pseudolite receivers (NovAtel Millennium) and two antennas. The two GPS antennas 
were mounted in the aircraft head cone. One was upwardlooking and the other downward-looking to track GPS and pseudolite signals. The raw GPS carrier phase measurements from the receivers were processed using an in-house software package; a modified version of the AIMSTM navigation processing software (Lee et al., 2003).

The measurement accuracy cannot be directly evaluated in the kinematic mode without an accurate reference trajectory. Alternatively, a comparison with the independent trajectory obtained by carrier phase dualfrequency DGPS post-processing using the GrafNav/GrafNet software and the double-differenced (DD) residuals computed from GPS/PL-predicted rover GPS antenna positions are used to analyse the performance of different models of pseudolite tropospheric delay.

For a short baseline, the double difference of carrier phase measurement in units of meters can be modelled as

$$
\nabla \Delta \Phi=\nabla \Delta \rho+\Delta d_{\text {trop }}^{P L}-\Delta d_{\text {trop }}^{G P S}+\lambda \nabla \Delta N
$$

The single-differenced pseudolite troposphere delay can be computed based on the double difference of carrier phase measurement (Fukushima et al., 2004)

$$
\nabla \Delta \Phi-\nabla \Delta \rho-\Delta d_{\text {trop }}^{P L}+\Delta d_{\text {trop }}^{G P S}=\lambda \nabla \Delta N
$$

As the GPS troposphere delay is modelled accurately for high elevation angles, $\Delta d_{\text {trop }}^{G P S}$ is treated errorless for the reference GPS satellite. The integer ambiguity is fixed if no cycle slip occurs. If no cycle slip occurred, the left side of Equation (11) should approximately maintain the same value as in the top of Figure 8, which is the carrier phase double difference of two GPS satellites. It is noticed that the double difference values have bias from an integer of cycle, which may be due to multipath effects.
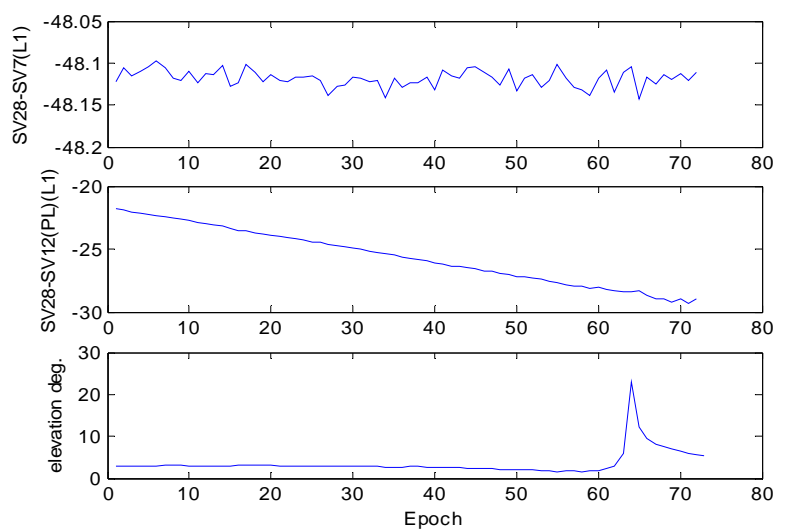

Fig. 8 Carrier phase double difference results and elevation angles
The middle of Figure 8 shows the double differences of the pseudolite and the reference satellite (SV28) without tropspheric delay applied to pseudolite measurements. It has a big drift (about 8 cycles) from the first to the last epoch even though no cycle slip occurred. This indicates that pseudolite tropospheric delay modelling affects the double difference results heavily. The bottom of Figure 8 depicts the elevation angle from the pseudolite to the rover receiver, which is an important parameter for the Saastamoinen and NMF models using the singledifferenced method.

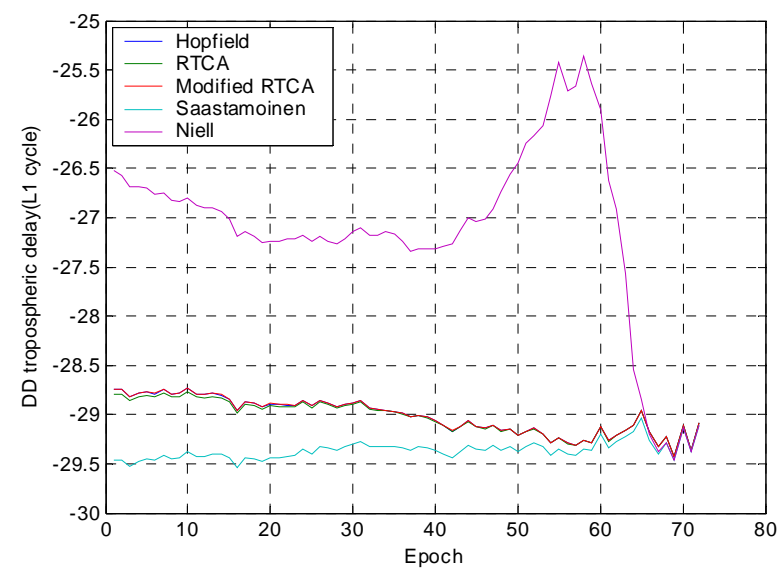

Fig. 9 Carrier phase double difference results of various tropospheric delay models

Figure 9 shows the carrier phase double difference results by applying the pseudolite tropospheric delay models introduced above. The results of the Saastamoinen model vary violently during most epochs. This means that the modelling is inaccurate. As shown in the bottom of Figure 8, the elevation angles from the pseudolite to the rover receiver are less than 5 degrees during most epochs, except the last few ones. The simulation results in Section 3 indicate that the Saastamoinen model cannot estimate correctly below 10 degrees. The results of the Saastamoinen model in the last few epochs become close to other models as the elevation angles increasing.

The results of RTCA, modified RTCA and Hopfield models are almost the same in Figure 9. This agrees with the simulation results in Figure 2, where the estimations of these models are the same when the elevation angle is zero.

The result of the NMF model is the best one among all the models tested as it almost keeps the same value in Figure 9, even if the elevation angle is very small in the flight test. This also agrees with the simulation results in Figures 2 and 3.

Compared to the GPS DD results in the top of Figure 8, the GPS $\backslash$ PL DD results in Figure 9 are more fluctuating. This may be due to the serious multipath effect of pseudolite signals, which is not only sensitive to the 
attitude of the aircraft as for GPS signals, but also the relative positions of the aircraft and the pseudolite.

\section{Closing Remarks}

This research reveals the importance of the tropospheric delay modelling for pseudolite positioning, and presents the performances of the six models proposed for pseudolite tropospheric delay estimation. Simulations were conducted for comparing their performances from different aspects and flight test data were processed to verify the simulation results.

It can be concluded from the simulation results that there are some differences between the tropospheric delays estimated with different models. Each model has its strength and weakness. The TRCA and modified TRCA models can be used in the applications with small height differences, such as aircraft landing and land-based applications. However, they are not suitable for these with large height difference, such as precise airborne georeferencing. Niell and Saastamoinen models are reliable when the elevation is above their limited elevation angles. The Hopfield and Bouska models perform relatively stable for the whole range of elevation angle though there is a reference height dependent bias between them. The Bouska model should have the smallest bias among all the models in the whole range of elevation angle.

The flight test results conform some of the conclusions from the simulation results. The single-differenced method proposed in this paper is effective to estimate the pseudolite tropospheric delay by employing GPS tropospheric models. It is found that the result of the NMF model is the best one among all the models tested even if the elevation angle is very small in the flight test. However, as the flight test data does not cover the range used in the simulation, the other conclusions from the simulation results should be further tested.

\section{Acknowledgements}

This study is supported by Australia Research Council Linkage project LP0349297: "Integration of GPS/PL/INS to Geo-reference Airborne Surveying and Mapping Sensors".

\section{References}

Biberger R J.; Teuber A.; Pany T.; Hein G W. (2003): Development of an APL Error Model for Precision Approaches and Validation by Flight Experiments. In: ION (Editor), GPS/GNSS, Portland, OR, pp. 2308-2317.

Bouska C T J.; Raquet J F. (2003): Tropospheric Model Error Reduction in Pseudolite Based Positioning Systems, ION GPS/GNSS 2003, Portland OR, USA, pp. 390-298.

Fukushima S.; Yoshihara T.; Suga S. (2004): Evaluation of a Tropospheric Correction Model fpr Airport Pseudolite, ION GPS/GNSS 2004, Long beach CA, USA.

Hofmann-Wellenhof B.; Lichtenegger H.; Collins J. (2000): GPS Theory and Practice. Fifth revised edition. SpringerVerlag Wien, New York, 382 pp.

Lee H K.; Wang J.; Rizos C.; Park W (2003): Carrier phase processing issues for high accuracy integrated GPS/Pseudolite/INS systems, Proceedings of 11th IAIN World Congress, Berlin, Germany, paper 252.

Niell A E. (1996): Global mapping functions for the atmosphere delay at radio wavelengths. Journal of Geophysical Research, 101(B2): 3227-3246.

RTCA (2000): GNSS Based Precision Approach Local Area Augmentation System (LAAS)-Signal-in-Space Interface Control Document (ICD). RTCA/DO-246A, Radio Technical Commission for Aeronautics.

Rueger J M. (1996): Electronic Distance Measurement, An Introduction. Springer, $276 \mathrm{pp}$. 